University of South Florida

DIGITAL COMMONS

Digital Commons @ University of

@ UNIVERSITY OF SOUTH FLORIDA

South Florida

USF Accountability Reports

USF Archives

$1-1-2019$

\title{
2019 Accountability Plan Tampa
}

USF

Follow this and additional works at: https://digitalcommons.usf.edu/usf_accountability_reports

\section{Scholar Commons Citation}

USF, "2019 Accountability Plan Tampa" (2019). USF Accountability Reports. 76.

https://digitalcommons.usf.edu/usf_accountability_reports/76

This Article is brought to you for free and open access by the USF Archives at Digital Commons @ University of South Florida. It has been accepted for inclusion in USF Accountability Reports by an authorized administrator of Digital Commons @ University of South Florida. For more information, please contact digitalcommons@usf.edu. 


\section{9 \\ Accountability Plan}
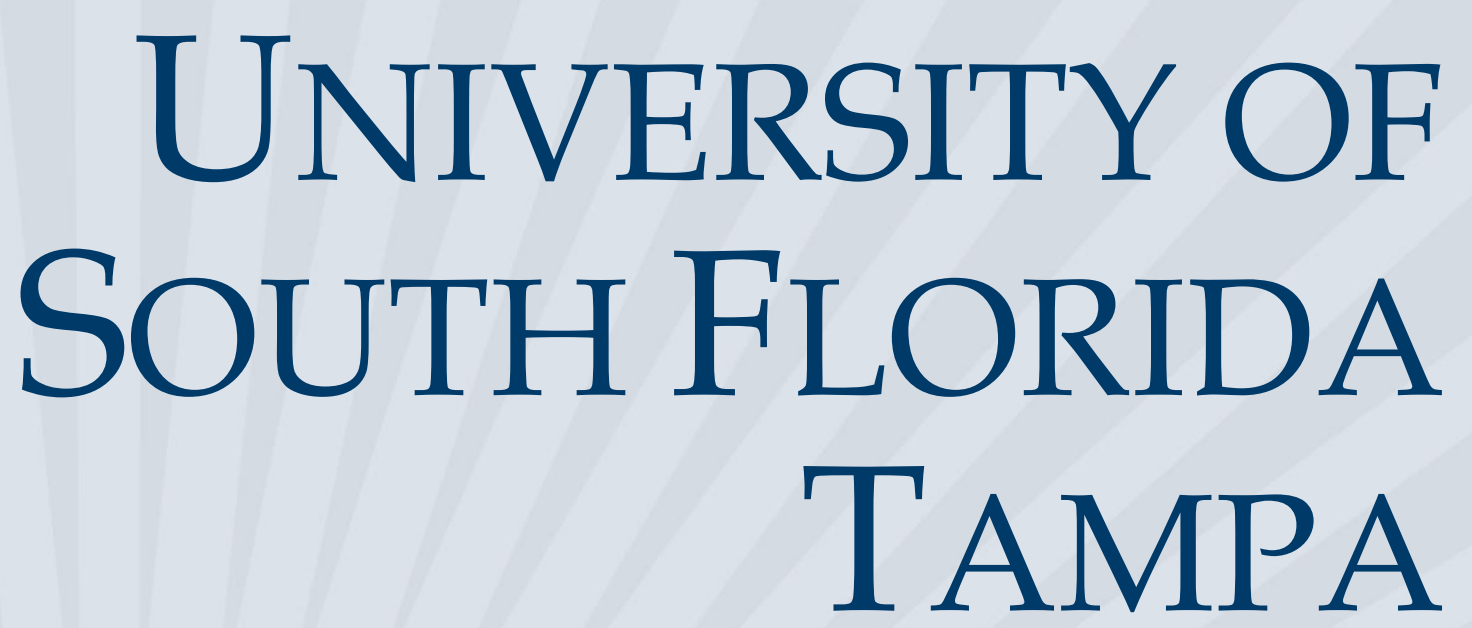

FINAL FOR BOG SUBMISSION

STATE UNIVERSITY SYSTEM of FLORIDA Board of Governors 


\section{INTRODUCTION}

This is a new report that combines the previous Annual Accountability Report and University Work Plans into a single document more closely aligned with the Board of Governors' 2025 System Strategic Plan.

This revised document will enhance the System's commitment to accountability and strategic planning by enabling comparisons between past goals and actual data to better assess performance, helping to foster greater coordination between institutional administrators, University Boards of Trustees, and the Board of Governors.

Once an Accountability Plan is approved by each institution's respective Boards of Trustees, the Board of Governors will review and consider the plan for approval, excluding those sections of the Plan that require additional regulatory or procedural approval pursuant to law or Board regulations. 


\section{TABLE OF CONTENTS}

1. PERFORMANCE BASED FUNDING METRICS, p. 3-4

2. PREEMINENT RESEARCH UNIVERSITY METRICS, p. 5-7

3. KEY PERFORMANCE INDICATORS

a. Teaching \& Learning, p. 8-10

b. Scholarship, Research and Innovation Metrics, p. 11-12

c. Institution Specific Goals, p. 12

4. ENROLLMENT PLANNING, p. 13-14 


\section{PERFORMANCE BASED FUNDING METRICS}

\begin{tabular}{|c|c|c|c|c|c|c|c|c|c|}
\hline & 2012-13 & 2013-14 & 2014-15 & 2015-16 & 2016-17 & 2017-18 & 2018-19 & $2019-20$ & $2020-21$ \\
\hline ACTUAL & 64.9 & 65.8 & 69.6 & 69.5 & 69.4 & . & . & . & . \\
\hline APPROVED GOALS & . & . & 66.8 & 70.0 & 70.5 & 71.5 & 72.5 & 73.0 & . \\
\hline PROPOSED GOALS & . & & . & 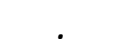 & . & 71.5 & 72.5 & 73.0 & 74.0 \\
\hline
\end{tabular}

2. Median Wages of Bachelor's Graduates Employed Full-time

\begin{tabular}{cccccccccc} 
& $2012-13$ & $2013-14$ & $2014-15$ & $2015-16$ & $2016-17$ & $2017-18$ & $2018-19$ & $2019-20$ & $2020-21$ \\
\hline ACTUAL & 35,300 & 36,500 & 38,000 & 37,400 & 38,100 &. &. &. & \\
APPROVED GOALS &. &. &. & 38,500 & 38,000 & 39,000 & 40,000 & 40,700 &. \\
PROPOSED GOALS &. &. &. &. &. & 39,000 & 40,700 & 41,200 & 41,700
\end{tabular}

3. Average Cost to the Student [Net Tuition \& Fees per 120 Credit Hours for Resident Undergraduates] $\begin{array}{lllllllll}2013-14 & 2014-15 & 2015-16 & 2016-17 & 2017-18 & 2018-19 & 2019-20 & 2020-21 & 2021-22\end{array}$ ACTUAL APPROVED GOALS Data Reported at System Level

PROPOSED GOALS

\section{FTIC Fourr-Year Graduation Rate [Full-time only]}

\begin{tabular}{|c|c|c|c|c|c|c|c|c|c|}
\hline & 2010-14 & 2011-15 & $2012-16$ & 2013-17 & 2014-18 & 2015-19 & 2016-20 & 2017-21 & 2018-22 \\
\hline $\begin{array}{l}\text { ACTUAL Graduated } \\
\text { Within USF System }\end{array}$ & 44.6 & 51.4 & 55.2 & 59.8 & 60.9 & . & . & . & . \\
\hline $\begin{array}{l}\text { ACTUAL Graduated } \\
\text { Within Same Campus }\end{array}$ & 44.3 & 50.9 & 55.1 & 59.7 & 60.5 & . & . & . & . \\
\hline APPROVED GOALS & . & . & . & 56.0 & 60.0 & 62.0 & 64.0 & 64.0 & . \\
\hline PROPOSED GOALS & . & . & . & . & . & 62.0 & 64.0 & 65.0 & 67.5 \\
\hline
\end{tabular}

5. Academic Progress Rate [Second Year Retention Rate with At Least a 2.0 GPA]

\begin{tabular}{|c|c|c|c|c|c|c|c|c|c|}
\hline & 2013-14 & 2014-15 & 2015-16 & 2016-17 & 2017-18 & 2018-19 & $2019-20$ & $2020-21$ & 2021-22 \\
\hline $\begin{array}{l}\text { ACTUAL Graduated } \\
\text { Within USF System }\end{array}$ & 87.0 & 87.1 & 88.0 & 87.4 & 88.8 & . & . & . & • \\
\hline $\begin{array}{l}\text { ACTUAL Graduated } \\
\text { Within Same Campus }\end{array}$ & 86.7 & 85.6 & 87.9 & $87.6 *$ & 88.7 & . & . & . & . \\
\hline APPROVED GOALS & . & . & 86.5 & 88.0 & 89.0 & 90.0 & 90.0 & 90.5 & . \\
\hline PROPOSED GOALS & . & . & . & . & . & 90.0 & 90.5 & 91.0 & 91.5 \\
\hline
\end{tabular}

Note*: Previous year data updated to reflect the change in methodology made by Board ODA staff to improve accuracy.

Note: Metrics are defined in appendix. For more information about the PBF model visit: http://www.flbog.edu/about/budget/performance_funding.php. 


\begin{tabular}{cccccccccc} 
6. Percentage of Bachelor's Degrees Awarded within Programs of Strategic Emphasis \\
& $2013-14$ & $2014-15$ & $2015-16$ & $2016-17$ & $2017-18$ & $2018-19$ & $2019-20$ & $2020-21$ & $2021-22$ \\
\hline ACTUAL & 53.5 & 56.6 & 62.0 & 64.4 & 64.5 &. &. &. &. \\
APPROVED GOALS &. &. & 57.0 & 62.0 & 64.5 & 65.0 & 65.5 & 66.0 &. \\
PROPOSED GOALS &. &. &. &. &. & 65.0 & 66.0 & 67.0 & 68.0
\end{tabular}

7. University Access Rate [Percent of Undergraduates with a Pell grant]

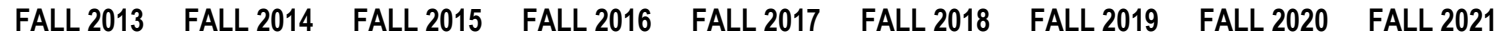

\begin{tabular}{ccccccccccc}
\hline ACTUAL & 42.5 & 42.8 & 40.9 & 39.9 & 41.6 &. &. &. &. \\
APPROVED GOALS &. &. & 40.0 & 40.0 & 40.0 & 40.0 & 40.0 & 40.0 &. \\
PROPOSED GOALS &. &. &. &. &. & 42.0 & 42.0 & 42.0 & 42.0
\end{tabular}

8. Percentage of Graduate Degrees Awarded within Programs of Strategic Emphasis

\begin{tabular}{cccccccccc} 
& $2013-14$ & $2014-15$ & $2015-16$ & $2016-17$ & $2017-18$ & $2018-19$ & $2019-20$ & $2020-21$ & $2021-22$ \\
\hline ACTUAL & 71.5 & 76.3 & 78.0 & 78.6 & 76.5 &. &. &. &. \\
APPROVED GOALS &. &. & 76.3 & 78.0 & 78.9 & 79.0 & 79.0 & 79.5 &. \\
PROPOSED GOALS &. &. &. &. &. & 77.0 & 77.0 & 77.0 & 77.0
\end{tabular}

9. BOG Choice: Percent of Baccalaureate Degrees Awarded Without Excess Hours

\begin{tabular}{cccccccccc} 
& $2013-14$ & $2014-15$ & $2015-16$ & $2016-17$ & $2017-18$ & $2018-19$ & $2019-20$ & $2020-21$ & $2021-22$ \\
\hline ACTUAL & 62.8 & 65.1 & 75.5 & 78.4 & 80.6 &. &. &. &. \\
APPROVED GOALS &. &. & 67.5 & 77.0 & 79.0 & 79.5 & 80.0 & 80.0 &. \\
PROPOSED GOALS &. &. &. &. &. & 81.5 & 81.5 & 82.5 & 83.0
\end{tabular}

\subsection{Current BOT Choice: Number of Postdoctoral Appointees}

$\begin{array}{lllllllll}\text { FALL } 2013 & \text { FALL } 2014 & \text { FALL } 2015 & \text { FALL } 2016 & \text { FALL } 2017 & \text { FALL } 2018 & \text { FALL } 2019 & \text { FALL 2020 } & \text { FALL } 2021\end{array}$

\begin{tabular}{ccccccccccc}
\hline ACTUAL & 321 & 300 & 277 & 267 & $276^{*}$ &. &. &. &. \\
APPROVED GOALS &. &. & 277 & 267 & 260 & 260 & 260 & 260 &. \\
PROPOSED GOALS &. &. &. &. &. & 308 & 308 & 308 & 308
\end{tabular}

Note: Revised since reported to NSF.

\begin{tabular}{|c|c|c|c|c|c|c|c|c|c|}
\hline & 2008-14 & 2009-15 & 2010-16 & 2011-17 & 2012-18 & 2013-19 & $2014-20$ & 2015-21 & 2016-22 \\
\hline $\begin{array}{l}\text { ACTUAL Graduated } \\
\text { Within USF System }\end{array}$ & 66.9 & 68.5 & 67.7 & 71.2 & 73.8 & . & . & . & . \\
\hline ACTUAL & 66.6 & 68.1 & 67.3 & 70.6 & 73.1 & . & . & $\cdot$ & . \\
\hline APPROVED GOALS & . & . & 66.3 & 71.0 & 73.0 & 75.0 & 77.0 & 78.0 & . \\
\hline PROPOSED GOALS & . & 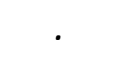 & . & . & . & 74.8 & 74.8 & 75.2 & 76.5 \\
\hline
\end{tabular}

Note: This is a transition year for the BOT Choice metric $(\# 10)$, so we are reporting data for both the current and future metrics. Metrics are defined in appendix. For more information about the PBF model visit: http://www.flbog.edu/about/budget/performance funding.php 


\section{PREEMINENT RESEARCH UNIVERSITY FUNDING METRICS (USF-TAMPA ONLY)}

\section{1a. Average GPA}

\begin{tabular}{|c|c|c|c|c|c|c|c|c|c|}
\hline & Fall 2014 & Fall 2015 & Fall 2016 & Fall 2017 & Fall 2018 & Fall 2019 & Fall 2020 & Fall 2021 & Fall 2022 \\
\hline ACTUAL & 4.0 & 4.1 & 4.1 & 4.1 & 4.1 & . & . & . & . \\
\hline APPROVED GOALS & . & . & 4.0 & 4.1 & 4.1 & 4.1 & 4.1 & 4.1 & . \\
\hline PROPOSED GOALS & . & . & . & . & . & 4.1 & 4.1 & 4.1 & 4.1 \\
\hline \multicolumn{10}{|c|}{ 1b. Average SAT Score* } \\
\hline & Fall 2014 & Fall 2015 & Fall 2016 & Fall 2017 & Fall 2018 & Fall 2019 & Fall 2020 & Fall 2021 & Fall 2022 \\
\hline ACTUAL & $1197^{*}$ & $1223^{*}$ & $1226 *$ & $1280^{*}$ & 1296 & . & . & $\cdot$ & . \\
\hline APPROVED GOALS & . & . & 1220 & 1280 & 1282 & 1285 & 1290 & 1290 & . \\
\hline PROPOSED GOALS & . & . & . & . & . & 1296 & 1300 & 1302 & 1305 \\
\hline \multicolumn{10}{|c|}{ Note*: Historical scores and approved goals were based upon a different SAT scale standard. } \\
\hline \multicolumn{10}{|c|}{ 2. Public University National Ranking [Top50 rankings based on BOG's official list of publications] } \\
\hline & 2015 & 2016 & 2017 & 2018 & 2019 & 2020 & 2021 & 2022 & 2023 \\
\hline ACTUAL & 3 & 4 & 4 & 4 & 4 & . & . & . & . \\
\hline APPROVED GOALS & . & . & 3 & 5 & 5 & 5 & 5 & 5 & . \\
\hline PROPOSED GOALS & . & . & . & . & . & 5 & 5 & 5 & 5 \\
\hline
\end{tabular}

3. Freshman Retention Rate [Full-time students as reported to IPEDS]

\begin{tabular}{cccccccccc} 
& $\mathbf{2 0 1 3 - 1 4}$ & $\mathbf{2 0 1 4 - 1 5}$ & $\mathbf{2 0 1 5 - 1 6}$ & $\mathbf{2 0 1 6 - 1 7}$ & $\mathbf{2 0 1 7 - 1 8}$ & $\mathbf{2 0 1 8 - 1 9}$ & $\mathbf{2 0 1 9 - 2 0}$ & $\mathbf{2 0 2 0 - 2 1}$ & $\mathbf{2 0 2 1 - 2 2}$ \\
\hline ACTUAL & 89 & 88 & 90 & 90 & 91 &. &. &. &. \\
APPROVED GOALS &. &. & 90 & 91 & 91 & 91 & 92 & 92 &. \\
PROPOSED GOALS &. &. &. &. &. & 91 & 92 & 93 & 93
\end{tabular}

Note: Metrics are defined in appendix. For more information about the PBF model visit: http://www.flbog.edu/about/budget/performance_funding.php 


\section{PREEMINENT RESEARCH UNIVERSITY FUNDING METRICS (CONT.) (USF TAMPA ONLY)}

4. Fourr-year Graduation Rate [Full-time students only]

\begin{tabular}{cccccccccc} 
& $\mathbf{2 0 1 0 - 1 4}$ & $\mathbf{2 0 1 1 - 1 5}$ & $\mathbf{2 0 1 2 - 1 6}$ & $\mathbf{2 0 1 3 - 1 7}$ & $\mathbf{2 0 1 4 - 1 8}$ & $\mathbf{2 0 1 5 - 1 9}$ & $\mathbf{2 0 1 6 - 2 0}$ & $\mathbf{2 0 1 7 - 2 1}$ & $\mathbf{2 0 1 8 - 2 2}$ \\
\hline ACTUAL & 44 & 51 & 55 & 60 & 61 &. &. &. &. \\
APPROVED GOALS &. &. & 50 & 56 & 60 & 62 & 64 & 64 &. \\
PROPOSED GOALS &. &. &. &. &. & 62 & 64 & 65 & 67.5
\end{tabular}

\section{National Academy Memberships}

\begin{tabular}{cccccccccc} 
& 2015 & 2016 & 2017 & 2018 & 2019 & $\mathbf{2 0 2 0}$ & $\mathbf{2 0 2 1}$ & $\mathbf{2 0 2 2}$ & 2023 \\
\hline ACTUAL & 9 & 8 & 11 & 13 & 14 &. &. &. &. \\
APPROVED GOALS &. &. & 9 & 10 & 13 & 13 & 13 & 13 &. \\
PROPOSED GOALS &. &. &. &. &. & 14 & 15 & 16 & 17
\end{tabular}

6. Science \& Engineering Research Expenditures (\$M)

\begin{tabular}{cccccccccc} 
& $2013-14$ & $2014-15$ & $2015-16$ & $2016-17$ & $2017-18$ & $\mathbf{2 0 1 8 - 1 9}$ & $\mathbf{2 0 1 9 - 2 0}$ & $\mathbf{2 0 2 0 - 2 1}$ & $\mathbf{2 0 2 1 - 2 2}$ \\
\hline ACTUAL & 438 & 420 & 448 & 502 & 525 &. &. &. &. \\
APPROVED GOALS &. &. & 421 & 427 & 503 & 504 & 505 & 506 &. \\
PROPOSED GOALS &. &. &. &. &. & 530 & 545 & 560 & 575
\end{tabular}

7. Non-Medical Science \& Engineering Research Expenditures (\$M)

\begin{tabular}{cccccccccc} 
& $2013-14$ & $2014-15$ & $2015-16$ & $2016-17$ & $\mathbf{2 0 1 7 - 1 8}$ & $\mathbf{2 0 1 8 - 1 9}$ & $\mathbf{2 0 1 9 - 2 0}$ & $\mathbf{2 0 2 0 - 2 1}$ & $\mathbf{2 0 2 1 - 2 2}$ \\
\hline ACTUAL & 239 & 229 & 229 & 288 & 296 &. &. &. &. \\
APPROVED GOALS &. &. & 230 & 233 & 289 & 290 & 291 & 292 &. \\
PROPOSED GOALS &. &. &. &. &. & 296 & 300 & 305 & 310
\end{tabular}

8. Number of Broad Disciplines Ranked in Top 100 for Research Expenditures

\begin{tabular}{cccccccccc} 
& $2012-13$ & $2013-14$ & $2014-15$ & $2015-16$ & $2016-17$ & $2017-18$ & $2018-19$ & $2019-20$ & $2020-21$ \\
\hline ACTUAL & 7 of 8 & 7 of 8 & 7 of 8 & 7 of 8 & 7 of 8 &. &. &. & \\
APPROVED GOALS &. &. & 7 of 8 & 8 of 8 & 8 of 8 & 8 of 8 & 8 of 8 & 8 of 8 &. \\
PROPOSED GOALS &. &. &. &. &. & 8 of 8 & 8 of 8 & 8 of 8 & 8 of 8
\end{tabular}

Note: Metrics are defined in appendix. For more information about the PBF model visit:

http://www.flbog.edu/about/budget/performance_funding.php 


\section{PREEMINENT RESEARCH UNIVERSITY FUNDING METRICS (CONT.) (USF TAMPA ONLY)}

9. Utility Patents Awarded [over three calendar years]

\begin{tabular}{cccccccccc} 
& $2012-14$ & $2013-15$ & $2014-16$ & $2015-17$ & $2016-18$ & $2017-19$ & $\mathbf{2 0 1 8 - 2 0}$ & $\mathbf{2 0 1 9 - 2 1}$ & $\mathbf{2 0 2 0 - 2 2}$ \\
\hline ACTUAL & 291 & 297 & 314 & 324 & 335 &. &. &. &. \\
APPROVED GOALS &. &. & 291 & 273 & 325 & 325 & 325 & 325 &. \\
PROPOSED GOALS &. &. &. &. &. & 323 & 306 & 309 & 312
\end{tabular}

10. Doctoral Degrees Awarded Annually

\begin{tabular}{cccccccccc} 
& $2013-14$ & $2014-15$ & $2015-16$ & $2016-17$ & $2017-18$ & $2018-19$ & $2019-20$ & 2020-21 & 2021-22 \\
\hline ACTUAL & 546 & 601 & 704 & 721 & 702 &. &. &. & \\
APPROVED GOALS &. &. & 645 & 650 & 725 & 730 & 735 & 740 &. \\
PROPOSED GOALS &. &. &. &. &. & 717 & 720 & 725 & 725
\end{tabular}

\section{Number of Post-Doctoral Appointees*}

\begin{tabular}{ccc|c|cccccc} 
& Fall 2013 & Fall 2014 & $\begin{array}{c}\text { Fall 2015 } \\
\text { OFFICIAL }\end{array}$ & Fall 2016 & Fall 2017 & Fall 2018 & Fall 2019 & Fall 2020 & Fall 2020 \\
\hline ACTUAL & 321 & 300 & 277 & 267 & $276^{*}$ &. &. &. &. \\
APPROVED GOALS & 321 & 300 & 277 & 267 & 260 & 260 &. &. &. \\
PROPOSED GOALS &. &. &. &. &. & 308 & 308 & 308 & 308
\end{tabular}

Note*: There is a time lag for the count of Post-Doctoral Appointees because statute requires that this data is as reported by the Center for Measuring University Performance in their annual Top American Research Universities (TARU) report. Revised since reported to NSF. Modified non-substantive edit post-submission.

\section{Endowment Size (\$Millions)}

\begin{tabular}{cccccccccc} 
& $2013-14$ & $2014-15$ & $2015-16$ & $\mathbf{2 0 1 6 - 1 7}$ & $\mathbf{2 0 1 7 - 1 8}$ & $\mathbf{2 0 1 8 - 1 9}$ & $\mathbf{2 0 1 9 - 2 0}$ & $\mathbf{2 0 2 0 - 2 1}$ & $\mathbf{2 0 2 1 - 2 2}$ \\
\hline ACTUAL & 417 & 417 & 395 & 442 & 480 &. &. &. &. \\
APPROVED GOALS &. &. & 395 & 412 & 450 & 465 & 485 & 500 &. \\
PROPOSED GOALS &. &. &. &. &. & 466 & 486 & 505 & 525
\end{tabular}

Note: Metrics are defined in appendix. For more information about the PBF model visit: http://www.flbog.edu/about/budget/performance funding.php 


\section{KEY PERFORMANCE INDICATORS}

Teaching \& Learning Metrics (from the 2025 System Strategic Plan that are not included in the PBF section) Public University National Ranking [Number of Top50 Rankings based on BOG's official list of publications]

\begin{tabular}{cccccccccc} 
& 2015 & 2016 & 2017 & 2018 & 2019 & 2020 & 2021 & 2022 & 2023 \\
\hline ACTUAL & 3 & 4 & 4 & 4 & 4 &. &. &. &. \\
APPROVED GOALS &. &. & 5 & 5 & 5 & 5 & 5 & 5 &. \\
PROPOSED GOALS &. &. &. &. &. & 5 & 5 & 5 & 5
\end{tabular}

Freshmen in Top 10\% of High School Class

Fall 2014 Fall 2015 Fall 2016 Fall 2017 Fall 2018 Fall 2019 Fall 2020 Fall 2021 Fall 2022

\begin{tabular}{|c|c|c|c|c|c|c|c|c|c|}
\hline ACTUAL & 33.6 & 36.3 & 35.1 & 37.1 & 34.2 & . & . & . & . \\
\hline APPROVED GOALS & . & . & 35.0 & 35.0 & 37.5 & 38.0 & 38.5 & 39.0 & . \\
\hline PROPOSED GOALS & . & . & . & . & . & 36.0 & 37.0 & 39.0 & 39.0 \\
\hline \multicolumn{10}{|c|}{ Time to Degree for FTICs in $120 \mathrm{hr}$ programs } \\
\hline & 2013-14 & 2014-15 & $2015-16$ & 2016-17 & 2017-18 & 2018-19 & $2019-20$ & $2020-21$ & 2021-22 \\
\hline ACTUAL & 4.9 & 4.8 & 4.3 & 4.2 & 4.0 & . & . & . & . \\
\hline APPROVED GOALS & . & . & 4.7 & 4.5 & 4.1 & 4.1 & 4.1 & 4.1 & . \\
\hline PROPOSED GOALS & . & . & . & . & . & 4.0 & 4.0 & 4.0 & 4.0 \\
\hline
\end{tabular}

Six-Year FTIC Graduation Rates [full-\& part-time students]

\begin{tabular}{|c|c|c|c|c|c|c|c|c|c|}
\hline & 2008-14 & $2009-15$ & $2010-16$ & 2011-17 & 2012-18 & 2013-19 & $2014-20$ & 2015-21 & 2016-22 \\
\hline $\begin{array}{l}\text { ACTUAL Graduated } \\
\text { Within USF System }\end{array}$ & 66.9 & 68.5 & 67.7 & 71.2 & 73.8 & . & . & & . \\
\hline $\begin{array}{l}\text { ACTUAL Graduated } \\
\text { Same Campus }\end{array}$ & 66.6 & 68.1 & 67.3 & 70.6 & 73.1 & . & . & . & . \\
\hline APPROVED GOALS & . & . & 66.3 & 71.0 & 73.0 & 75.0 & 77.0 & 78.0 & . \\
\hline PROPOSED GOALS & . & • & . & • & . & 74.8 & 74.8 & 75.2 & 76.5 \\
\hline
\end{tabular}

Bachelor's Degrees Awarded [First Majors Only]

\begin{tabular}{cccccccccc} 
& $2013-14$ & $2014-15$ & $2015-16$ & $2016-17$ & $2017-18$ & $2018-19$ & $2019-20$ & $2020-21$ & $2021-22$ \\
\hline ACTUAL & 8,079 & 7,991 & 7,876 & 8,101 & 8,295 &. &. &. &. \\
APPROVED GOALS &. &. & 7,700 & 7,900 & 8,100 & 8,150 & 8,150 & 8,200 &. \\
PROPOSED GOALS &. &. &. &. &. & 8,300 & 8,350 & 8,400 & 8,500
\end{tabular}




\section{KEY PERFORMANCE INDICATORS (CONTINUED)}

\section{Teaching \& Learning Metrics}

Professional Licensure \& Certification Exam First-time Pass Rates

\begin{tabular}{lccccccccc}
\multicolumn{1}{c}{ CALENDAR YEAR } & $\mathbf{2 0 1 4}$ & $\mathbf{2 0 1 5}$ & $\mathbf{2 0 1 6}$ & $\mathbf{2 0 1 7}$ & $\mathbf{2 0 1 8}$ & $\mathbf{2 0 1 9}$ & $\mathbf{2 0 2 0}$ & $\mathbf{2 0 2 1}$ & $\mathbf{2 0 2 2}$ \\
\hline Nursing & 86 & 90 & 94 & 93 & 94 & 93 & 100 & 100 & 100 \\
US Average & 85 & 87 & 85 & 87 & 92 &. &. &. &. \\
\hline Medicine $(2 Y \mathrm{Yr})$ & 95 & 96 & 94 & 92 & 98 & 98 & 100 & 100 & 100 \\
US Average & 96 & 95 & 96 & 96 & 96 &. &. &. &. \\
\hline Pharmacy &. & 94 & 91 & 86 & 83 & 90 & 100 & 100 & 100 \\
US Average & 95 & 93 & 86 & 88 & 89 & & & & GOAL \\
\hline
\end{tabular}

\begin{tabular}{|c|c|c|c|c|c|c|c|c|c|}
\hline CROSS-YEAR & 2013-14 & 2014-15 & 2015-16 & 2016-17 & 2017-18 & $\begin{array}{c}2018-19 \\
\text { GOAL }\end{array}$ & $\begin{array}{c}2019-20 \\
\text { GOAL }\end{array}$ & $\begin{array}{c}2020-21 \\
\text { GOAL }\end{array}$ & $\begin{array}{c}2021-22 \\
\text { GOAL }\end{array}$ \\
\hline Medicine (4Y-CK) & 98 & 97 & 99 & 95 & 98 & 98 & 100 & 100 & 100 \\
\hline US Average & 97 & 95 & 96 & 96 & 97 & . & . & . & . \\
\hline Medicine (4Y-CS) & 91 & 96 & 97 & 96 & 97 & 98 & 100 & 100 & 100 \\
\hline US Average & 96 & 96 & 97 & 96 & 95 & 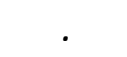 & . & & . \\
\hline MULTI-YEAR & 2012-14 & 2013-15 & 2014-16 & $2015-17$ & 2016-18 & $\begin{array}{c}2017-19 \\
\text { GOAL }\end{array}$ & $\begin{array}{c}2018-20 \\
\text { GOAL }\end{array}$ & $\begin{array}{c}2019-21 \\
\text { GOAL }\end{array}$ & $\begin{array}{c}2020-22 \\
\text { GOAL }\end{array}$ \\
\hline Physical Therapy & 97 & 95 & 94 & 95 & 94 & 93 & 100 & 100 & 100 \\
\hline US Average & 90 & 91 & 92 & 92 & 92 & . & . & r. & . \\
\hline
\end{tabular}

\section{Exam Scores Relative to Benchmarks}

\begin{tabular}{llllllllll}
\hline Above or Tied & 3 & 6 & 5 & 3 & 5 & 6 & 6 & 6 & 6 \\
Total & 5 & 6 & 6 & 6 & 6 & 6 & 6 & 6 & 6
\end{tabular}

Note: An asterisk $\left(^{*}\right)$ indicates the passing rate is preliminary. 


\section{KEY PERFORMANCE INDICATORS (CONTINUED)}

\section{Teaching \& Learning Metrics}

Graduate Degrees Awarded [First Majors Only]

\begin{tabular}{cccccccccc} 
& $\mathbf{2 0 1 3 - 1 4}$ & $\mathbf{2 0 1 4 - 1 5}$ & $\mathbf{2 0 1 5 - 1 6}$ & $\mathbf{2 0 1 6 - 1 7}$ & $\mathbf{2 0 1 7 - 1 8}$ & $\mathbf{2 0 1 8 - 1 9}$ & $\mathbf{2 0 1 9 - 2 0}$ & $\mathbf{2 0 2 0 - 2 1}$ & $\mathbf{2 0 2 1 - 2 2}$ \\
\hline ACTUAL & 3,179 & 3,501 & 3,654 & 3,914 & 3,878 &. &. &. & \\
APPROVED GOALS &. &. & 3,600 & 3,675 & 3,925 & 3,930 & 3,935 & 3,940 &. \\
PROPOSED GOALS &. &. &. &. &. & 3,888 & 3,898 & 3,908 & 3,918
\end{tabular}

Percent of Bachelor's Degrees Awarded to African-American \& Hispanic Students

\begin{tabular}{cccccccccc} 
& $2013-14$ & $2014-15$ & $2015-16$ & $2016-17$ & $2017-18$ & $\mathbf{2 0 1 8 - 1 9}$ & $\mathbf{2 0 1 9 - 2 0}$ & $\mathbf{2 0 2 0 - 2 1}$ & $\mathbf{2 0 2 1 - 2 2}$ \\
\hline ACTUAL & 31 & 32 & 33 & 34 & 32 &. &. &. &. \\
APPROVED GOALS &. &. & 31 & 33 & 34 & 34 & 34 & 34 &. \\
PROPOSED GOALS &. &. &. &. &. & 34 & 34 & 35 & 35
\end{tabular}

Percent of Adult (Aged 25+) Undergraduates Enrolled

\begin{tabular}{cccccccccc} 
& Fall 2014 & Fall 2015 & Fall 2016 & Fall 2017 & Fall 2018 & Fall 2019 & Fall 2020 & Fall 2021 & Fall 2022 \\
\hline ACTUAL & 21 & 20 & 19 & 19 & 18 &. &. &. &. \\
APPROVED GOALS &. &. & 20 & 19 & 18 & 18 & 18 & 18 &. \\
PROPOSED GOALS &. &. &. &. &. & 18 & 18 & 18 & 18
\end{tabular}

Percent of Undergraduate FTE in Online Courses

\begin{tabular}{cccccccccc} 
& $2013-14$ & $2014-15$ & $2015-16$ & $2016-17$ & $2017-18$ & $2018-19$ & $2019-20$ & $2020-21$ & $2021-22$ \\
\hline ACTUAL & 21 & 24 & 26 & 27 & 29 &. &. &. &. \\
APPROVED GOALS &. &. & 25.5 & 26.2 & 27.5 & 28.7 & 29.8 & 30.8 &. \\
PROPOSED GOALS &. &. &. &. &. & 30.0 & 31.0 & 31.0 & 33.0
\end{tabular}

Percent of Bachelor's Degrees in STEM \& Health

\begin{tabular}{cccccccccc} 
& $2013-14$ & $2014-15$ & $2015-16$ & $2016-17$ & $2017-18$ & $2018-19$ & $2019-20$ & $2020-21$ & $2021-22$ \\
\hline ACTUAL & 37 & 42 & 46 & 48 & 49 &. &. &. &. \\
APPROVED GOALS &. &. & 44 & 46 & 48 & 48 & 48 & 48 &. \\
PROPOSED GOALS &. &. &. &. &. & 50 & 53 & 54 & 54
\end{tabular}

Percent of Graduate Degrees in STEM \& Health

\begin{tabular}{cccccccccc} 
& $2013-14$ & $2014-15$ & $2015-16$ & $2016-17$ & $\mathbf{2 0 1 7 - 1 8}$ & $\mathbf{2 0 1 8 - 1 9}$ & $\mathbf{2 0 1 9 - 2 0}$ & $\mathbf{2 0 2 0 - 2 1}$ & $\mathbf{2 0 2 1 - 2 2}$ \\
\hline ACTUAL & 56 & 61 & 65 & 66 & 64 &. &. &. &. \\
APPROVED GOALS &. &. & 64 & 65 & 66 & 67 & 67 & 67 &. \\
PROPOSED GOALS &. &. &. &. &. & 65 & 66 & 67 & 67
\end{tabular}




\section{KEY PERFORMANCE INDICATORS (CONTINUED)}

Scholarship, Research and Innovation Metrics

National Academy Memberships

\begin{tabular}{ccccccccccc} 
& 2015 & 2016 & 2017 & $\mathbf{2 0 1 8}$ & $\mathbf{2 0 1 9}$ & $\mathbf{2 0 2 0}$ & $\mathbf{2 0 2 1}$ & $\mathbf{2 0 2 2}$ & $\mathbf{2 0 2 3}$ \\
\hline ACTUAL & 7 & 8 & 11 & 13 & 14 &. &. &. &. \\
APPROVED GOALS &. &. & 10 & 10 & 13 & 13 & 13 & 13 &. \\
PROPOSED GOALS &. &. &. &. &. & 14 & 15 & 16 & 17
\end{tabular}

Faculty Awards

\begin{tabular}{|c|c|c|c|c|c|c|c|c|c|}
\hline & Fall 2012 & Fall 2013 & Fall 2014 & Fall 2015 & Fall 2016 & Fall 2017 & Fall 2018 & Fall 2019 & Fall 2020 \\
\hline ACTUAL & 7 & 8 & 8 & 13 & 13 & . & . & . & . \\
\hline APPROVED GOALS & . & . & 7 & 8 & 9 & 10 & 11 & 11 & . \\
\hline PROPOSED GOALS & . & . & . & . & . & 12 & 12 & 12 & 13 \\
\hline \multicolumn{10}{|c|}{ Total Research Expenditures (\$M) } \\
\hline & 2013-14 & 2014-15 & 2015-16 & 2016-17 & 2017-18 & 2018-19 & $2019-20$ & 2020-21 & 2021-22 \\
\hline ACTUAL & 489 & 485 & 506 & 558 & 582 & . & . & . & . \\
\hline APPROVED GOALS & . & . & 486 & 501 & 559 & 560 & 561 & 562 & . \\
\hline PROPOSED GOALS & . & . & . & . & . & 585 & 590 & 595 & 600 \\
\hline
\end{tabular}

Percentage of Research Expenditures Funded from External Sources

\begin{tabular}{|c|c|c|c|c|c|c|c|c|c|}
\hline & 2013-14 & 2014-15 & 2015-16 & 2016-17 & 2017-18 & 2018-19 & 2019-20 & $2020-21$ & 2021-22 \\
\hline ACTUAL & 60 & 55 & 55 & 57 & 57 & . & . & . & . \\
\hline APPROVED GOALS & . & . & 56 & 57 & 58 & 59 & 60 & 60 & . \\
\hline PROPOSED GOALS & . & . & . & . & . & 59 & 60 & 60 & 60 \\
\hline \multicolumn{10}{|c|}{ Utility Patents Awarded [from the USPTO] } \\
\hline & 2014 & 2015 & 2016 & 2017 & 2018 & 2019 & 2020 & 2021 & 2022 \\
\hline ACTUAL & 110 & 90 & 114 & 120 & 101 & . & . & . & . \\
\hline APPROVED GOALS & . & . & . & 69 & 121 & 122 & 122 & 122 & . \\
\hline PROPOSED GOALS & . & . & . & . & . & 102 & 103 & 104 & 105 \\
\hline
\end{tabular}




\section{KEY PERFORMANCE INDICATORS (CONTINUED)}

\section{Scholarship, Research and Innovation Metrics}

\begin{tabular}{|c|c|c|c|c|c|c|c|c|c|c|}
\hline \multicolumn{11}{|c|}{ Number of Licenses/Options Executed Annually } \\
\hline & 2013-14 & 2014-15 & \multicolumn{2}{|c|}{ 2015-16 } & 2016-17 & 2017-18 & \multirow{2}{*}{$\begin{array}{c}\text { 2018-19 } \\
.\end{array}$} & \multirow{2}{*}{$\begin{array}{c}2019-20 \\
.\end{array}$} & \multirow{2}{*}{$\begin{array}{c}2020-21 \\
.\end{array}$} & \multirow{2}{*}{$\begin{array}{c}2021-22 \\
. \\
\end{array}$} \\
\hline ACTUAL & 91 & 119 & & & 122 & 127 & & & & \\
\hline APPROVED GOALS & . & . & & & 121 & 123 & 123 & 123 & 123 & . \\
\hline PROPOSED GOALS & . & . & & & . & . & 90 & 91 & 92 & 93 \\
\hline \multicolumn{11}{|c|}{ Number of Start-up Companies Created } \\
\hline & 2012-13 & 2013-14 & 2014-15 & 2015-16 & 2016-17 & 2017-18 & 2018-19 & 2019-20 & 2020-21 & 2021-22 \\
\hline ACTUAL & 9 & 11 & 11 & 9 & 10 & 10 & . & . & . & . \\
\hline APPROVED GOALS & . & . & 8 & 8 & 9 & 10 & 11 & 11 & 11 & . \\
\hline PROPOSED GOALS & . & . & . & . & . & . & 10 & 11 & 11 & 12 \\
\hline
\end{tabular}

\section{Institution Specific Goals}

To further distinguish the university's distinctive mission, the university may choose to provide additional metric goals that are based on the university's own strategic plan.

\section{Percent of Course Sections Offered via Distance and Blended Learning}

\begin{tabular}{|c|c|c|c|c|c|c|c|c|}
\hline $\begin{array}{l}2013-14 \\
\text { ACTUAL }\end{array}$ & $\begin{array}{l}2014-15 \\
\text { ACTUAL }\end{array}$ & $\begin{array}{l}2015-16 \\
\text { ACTUAL }\end{array}$ & $\begin{array}{l}2016-17 \\
\text { ACTUAL }\end{array}$ & $\begin{array}{l}2017-18 \\
\text { ACTUAL }\end{array}$ & $\begin{array}{l}2018-19 \\
\text { GOAL }\end{array}$ & $\begin{array}{l}2019-20 \\
\text { GOAL }\end{array}$ & $\begin{array}{l}2020-21 \\
\text { GOAL }\end{array}$ & $\begin{array}{l}2021-22 \\
\text { GOAL }\end{array}$ \\
\hline 11 & 12 & 12 & 14 & 16 & 17 & 18 & 19 & 20 \\
\hline \multicolumn{9}{|c|}{ 2. Federal Research Expenditures (\$M) } \\
\hline $\begin{array}{l}2013-14 \\
\text { ACTUAL }\end{array}$ & $\begin{array}{l}2014-15 \\
\text { ACTUAL }\end{array}$ & $\begin{array}{l}2015-16 \\
\text { ACTUAL }\end{array}$ & $\begin{array}{l}2016-17 \\
\text { ACTUAL }\end{array}$ & $\begin{array}{l}2017-18 \\
\text { ACTUAL }\end{array}$ & $\begin{array}{l}2018-19 \\
\text { GOAL }\end{array}$ & $\begin{array}{l}2019-20 \\
\text { GOAL }\end{array}$ & $\begin{array}{l}2020-21 \\
\text { GOAL }\end{array}$ & $\begin{array}{l}2021-22 \\
\text { GOAL }\end{array}$ \\
\hline 223 & 218 & 228 & 250 & 263 & 252 & 253 & 254 & 255 \\
\hline
\end{tabular}




\section{ENROLLMENT PLANNING}

Fall Headcount Enrollment by Student Level (for all degree-seeking students at all campuses)

\begin{tabular}{|c|c|c|c|c|c|c|c|c|c|}
\hline & 2014 & 2015 & 2016 & 2017 & 2018 & 2019 & 2020 & 2021 & 2022 \\
\hline \multicolumn{10}{|l|}{ UNDERGRADUATE } \\
\hline ACTUAL & 30,281 & 30,288 & 30,550 & 30,920 & 31,325 & . & . & . & \\
\hline APPROVED GOALS & . & . & . & 30,332 & 30,656 & 30,633 & 30,737 & 30,889 & \\
\hline PROPOSED GOALS & . & & . & . & . & 31,275 & 31,900 & 32,600 & 33,500 \\
\hline \multicolumn{10}{|l|}{ GRADUATE } \\
\hline ACTUAL & 9,905 & 9,984 & 10,248 & 10,853 & 10,776 & & . & . & \\
\hline APPROVED GOALS & . & . & . & 10,324 & 10,923 & 11,010 & 11,115 & 11,222 & . \\
\hline PROPOSED GOALS & . & & . & . & . & 10,817 & 10,866 & 10,915 & 10,990 \\
\hline
\end{tabular}

Fall Headcount Enrollment by Student Type (for all degree-seeking students at all campuses)

\begin{tabular}{lrrrrr|rrrr} 
& 2014 & 2015 & 2016 & 2017 & 2018 & 2019 & 2020 & 2021 & 2022 \\
& ACTUAL & ACTUAL & ACTUAL & ACTUAL & ACTUAL & PLAN & PLAN & PLAN & PLAN \\
\hline UNDERGRADUATE & & & & & & & & & \\
\hline FTIC & 15,664 & 15,824 & 15,943 & 15,961 & 16,387 & 16,787 & 17,300 & 17,800 & 18,500 \\
FCS AA Transfers & 7,195 & 7,095 & 7,295 & 7,430 & 7,538 & 7,238 & 7,400 & 7,650 & 7,900 \\
Other AA Transfers & 1,171 & 1,104 & 1,052 & 1087 & 1,071 & 1,000 & 1,000 & 1,000 & 1,000 \\
Post-Baccalaureates & 805 & 764 & 735 & 746 & 691 & 750 & 750 & 750 & 750 \\
Other Undergraduates & 5,446 & 5,501 & 5,525 & 5696 & 5,638 & 5,500 & 5,450 & 5,400 & 5,350 \\
Subtotal & $\mathbf{3 0 , 2 8 1}$ & $\mathbf{3 0 , 2 8 8}$ & $\mathbf{3 0 , 5 5 0}$ & $\mathbf{3 0 , 9 2 0}$ & $\mathbf{3 1 , 3 2 5}$ & $\mathbf{3 1 , 2 7 5}$ & $\mathbf{3 1 , 9 0 0}$ & $\mathbf{3 2 , 6 0 0}$ & $\mathbf{3 3 , 5 0 0}$ \\
GRADUATE & & & & & & & & & \\
\hline Master's & 6,300 & 6,446 & 6,568 & 6,974 & 6,827 & 6,850 & 6,875 & 6,900 & 6,925 \\
Research Doctoral & 2,226 & 2,229 & 2,333 & 2,443 & 2,461 & 2,475 & 2,495 & 2,515 & 2,535 \\
Professional Doctoral & 1,379 & 1,309 & 1,348 & 1,436 & 1,488 & 1,492 & 1,496 & 1,500 & 1,530 \\
Subtotal & $\mathbf{9 , 9 0 5}$ & $\mathbf{9 , 9 8 4}$ & $\mathbf{1 0 , 2 4 8}$ & $\mathbf{1 0 , 8 5 3}$ & $\mathbf{1 0 , 7 7 6}$ & $\mathbf{1 0 , 8 1 7}$ & $\mathbf{1 0 , 8 6 6}$ & $\mathbf{1 0 , 9 1 5}$ & $\mathbf{1 0 , 9 9 0}$ \\
\hline \multicolumn{1}{c}{ TOTAL } & $\mathbf{4 0 , 1 8 6}$ & $\mathbf{4 0 , 2 7 2}$ & $\mathbf{4 0 , 7 9 8}$ & $\mathbf{4 1 , 7 7 3}$ & $\mathbf{4 2 , 1 0 1}$ & $\mathbf{4 2 , 0 9 2}$ & $\mathbf{4 2 , 7 6 6}$ & $\mathbf{4 3 , 5 1 5}$ & $\mathbf{4 4 , 4 9 0}$ \\
\hline
\end{tabular}

Notes: This table reports the number of students enrolled at the university by student type categories. The student type for undergraduates is based on the Type of Student at Time of Most Recent Admission. The student type for graduates is based on the degree that is sought and the student CIP code. Does not include 'Unclassified' students who are not formally admitted into a degree program but are enrolled (e.g., dual enrolled high school students).

\section{Percent of Baccalaureate-Seeking Resident Undergraduates Who Earned 15+ Credit Hours} (Fall terms only)

\begin{tabular}{ccccccccccc} 
& 2014 & 2015 & 2016 & 2017 & 2018 & 2019 & 2020 & 2021 & 2022 \\
\hline ACTUAL & 26 & 24 & 22 & 23 & 21 &. &. &. &. \\
APPROVED GOALS &. &. &. &. &. &. &. &. &. & 23 \\
PROPOSED GOALS &. &. &. &. &. & 21 & 22 & 23 & 24
\end{tabular}




\section{ENROLLMENT PLANNING continued}

\begin{tabular}{|c|c|c|c|c|c|c|c|c|c|c|}
\hline & $\begin{array}{l}\text { 2013-14 } \\
\text { ACTUAL }\end{array}$ & $\begin{array}{l}2014-15 \\
\text { ACTUAL }\end{array}$ & $\begin{array}{l}\text { 2015-16 } \\
\text { ACTUAL }\end{array}$ & $\begin{array}{l}2016-17 \\
\text { ACTUAL }\end{array}$ & $\begin{array}{l}2017-18 \\
\text { ACTUAL }\end{array}$ & $\begin{array}{l}2018-19 \\
\text { PLAN }\end{array}$ & $\begin{array}{l}2019-20 \\
\text { PLAN }\end{array}$ & $\begin{array}{l}2020-21 \\
\text { PLAN }\end{array}$ & $\begin{array}{l}2021-22 \\
\text { PLAN }\end{array}$ & $\begin{array}{c}2022-23 \\
\text { PLAN }\end{array}$ \\
\hline \multicolumn{11}{|l|}{ RESIDENT } \\
\hline LOWER & 10,427 & 10,282 & 10,210 & 10,209 & 10,052 & 10,001 & 10,181 & 10,370 & 10,518 & 10,685 \\
\hline UPPER & 15,897 & 15,554 & 15,452 & 15,005 & 15,451 & 15,439 & 15,558 & 15,670 & 15,823 & 15,954 \\
\hline GRAD I & 4,266 & 4,199 & 3,997 & 3,785 & 3,889 & 3,862 & 3,863 & 3,866 & 3,870 & 3,880 \\
\hline GRAD ॥ & 1,264 & 1,277 & 1,198 & 1,217 & 1,264 & 1,284 & 1,290 & 1,295 & 1,302 & 1,309 \\
\hline TOTAL & 31,853 & 31,311 & 30,858 & 30,216 & 30,657 & 30,586 & 30,892 & 31,201 & 31,513 & 31,828 \\
\hline \multicolumn{11}{|c|}{ NON-RESIDENT } \\
\hline LOWER & 1,284 & 1,501 & 1,766 & 1,866 & 1,812 & 1,801 & 1,929 & 2,065 & 2,165 & 2,243 \\
\hline UPPER & 958 & 1,184 & 1,446 & 1,647 & 1,801 & 1,923 & 2,055 & 2,164 & 2,293 & 2,434 \\
\hline GRAD I & 1,336 & 1,631 & 1,953 & 2,112 & 2,100 & 2,012 & 2,022 & 2,031 & 2,049 & 2,072 \\
\hline 646 & 853 & 880 & 935 & 1,016 & 1,117 & 1,156 & 1,142 & 1,150 & 1,170 & 1,201 \\
\hline TOTAL & 4,431 & 5,197 & 6,099 & 6,640 & 6,829 & 6,892 & 7,148 & 7,410 & 7,677 & 7,950 \\
\hline \multicolumn{11}{|l|}{ TOTAL } \\
\hline LOWER & 11,710 & 11,783 & 11,976 & 12,075 & 11,864 & 11,802 & 12,110 & 12,435 & 12,683 & 12,928 \\
\hline UPPER & 16,854 & 16,738 & 16,898 & 16,652 & 17,252 & 17,362 & 17,613 & 17,834 & 18,116 & 18,388 \\
\hline GRAD I & 5,603 & 5,830 & 5,950 & 5,897 & 5,989 & 5,874 & 5,885 & 5,897 & 5,919 & 5,952 \\
\hline GRAD ॥ & 2,116 & 2,157 & 2,132 & 2,232 & 2,381 & 2,440 & 2,432 & 2,445 & 2,472 & 2,510 \\
\hline TOTAL & 36,284 & 36,508 & 36,957 & 36,856 & 37,486 & 37,478 & 38,040 & 38,611 & 39,190 & 39,778 \\
\hline
\end{tabular}

Note: Full-time Equivalent (FTE) student is a measure of all instructional activity (regardless of fundability) that is based on the number of credit hours that degreeseeking students enroll. FTE is based on the standard national definition, which divides undergraduate credit hours by 30 and graduate credit hours by 24 . Pursuant to section 1013.31, Florida Statutes, Board facilities staff use this data as a key factor in the calculation of facility space needs for university educational plant surveys.

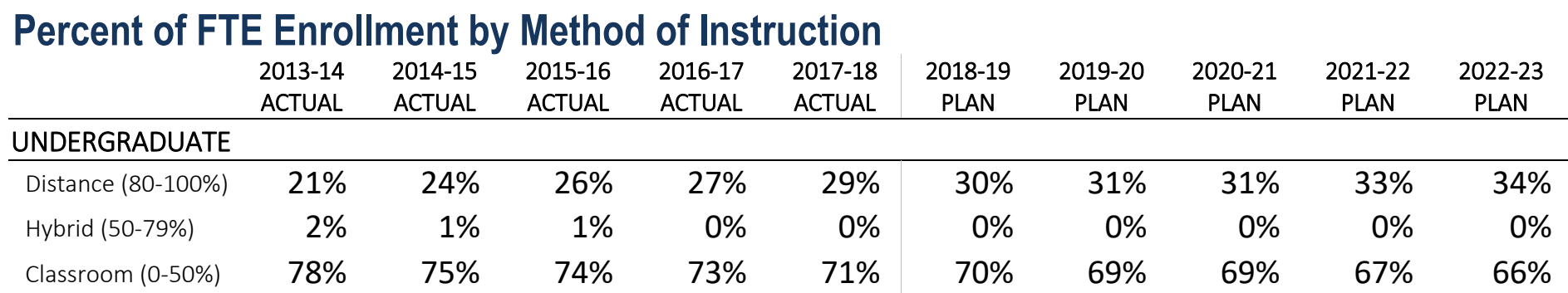

\section{GRADUATE}

\begin{tabular}{|c|c|c|c|c|c|c|c|c|c|c|}
\hline Distance (80-100\%) & $21 \%$ & $23 \%$ & $23 \%$ & $25 \%$ & $28 \%$ & $29 \%$ & $30 \%$ & $31 \%$ & $31 \%$ & $32 \%$ \\
\hline Hybrid (50-79\%) & $3 \%$ & $2 \%$ & $1 \%$ & $1 \%$ & $2 \%$ & $2 \%$ & $2 \%$ & $2 \%$ & $2 \%$ & $2 \%$ \\
\hline Classroom (0-50\%) & $76 \%$ & $75 \%$ & $76 \%$ & $73 \%$ & $70 \%$ & $69 \%$ & $68 \%$ & $67 \%$ & $66 \%$ & $66 \%$ \\
\hline
\end{tabular}

Note: Full-time Equivalent (FTE) student is a measure of instructional activity (regardless of fundability) that is based on the number of credit hours that degreeseeking students enroll. FTE is based on the standard national definition, which divides undergraduate credit hours by 30 and graduate credit hours by 24 . Distance Learning is a course in which at least 80 percent of the direct instruction of the course is delivered using some form of technology when the student and instructor are separated by time or space, or both (per 1009.24(17), F.S.). Classroom/Traditional, is a course in which less than 50\% of the direct instruction of the course is delivered using some form of technology when the student and instructor are separated by time, space or both. This designation can include activities that do not occur in a classroom (ie, labs, internships, practica, clinicals, labs, etc) - see SUDS data element \#2052. *Percentages may not total 100 due to rounding. 